\title{
AVALIAÇÃO DO USO DE FÁRMACOS EM ANIMAIS DE COMPANHIA SEM ORIENTAÇÃO PROFISSIONAL
}

\author{
ZIELKE, Marta ${ }^{1}$ \\ CARVALHO, Lianna Fernandes ${ }^{1}$; \\ SALAME, Jéssica Paola ${ }^{1}$; \\ BARBOZA, Daniele Vitor ${ }^{1}$; \\ GASPAR, Luiz Fernando Jantzen ${ }^{2}$; \\ SAMPAIO, Luzia Cristina Lencioni ${ }^{3}$.
}

Recebido: 03/03/2018

Aceito: 30/04/2018

\begin{abstract}
${ }^{1}$ Pós-graduanda do Programa de Residência Multiprofissional e em Área Profissional da Saúde/UFPEL;
${ }^{2}$ Professor do Departamento de Clínicas Veterinárias/UFPEL; ${ }^{3}$ Professora do Departamento de Clínicas Veterinárias/UFPEL.
\end{abstract}

\section{RESUMO}

$\mathrm{O}$ uso de fármacos em animais de companhia sem orientação profissional inclui a prescrição realizada por pessoas não qualificadas, o uso de formulações caseiras e a automedicação orientada - quando o tutor reutiliza prescrições antigas. Este trabalho visou determinar a prevalência dos casos de medicação em animais de companhia sem orientação do médico veterinário, bem como identificar os fármacos mais utilizados, estabelecendo dados loco regionais referentes aos usuários do Hospital de Clínicas Veterinárias da Universidade Federal de Pelotas (HCV/UFPEL), situado na cidade de Capão do Leão - RS. A pesquisa foi realizada a partir de questionário fechado disponibilizado em sala de espera do HCV/UFPEL, e as questões abordadas incluíram o uso de medicações comerciais ou naturais sem orientação do médico veterinário, a indicação de leigos e o perfil dos tutores. Foram coletados dados de 267 animais, sendo 198 caninos e 69 felinos, sob a responsabilidade de 180 tutores. Vinte e um por cento das mulheres e $19 \%$ dos homens medicam seus animais sem orientação profissional com regularidade. Setenta e dois por cento dos tutores afirmaram comunicar ao veterinário que o animal foi previamente medicado, e $9 \%$ já presenciaram toxicidade medicamentosa relacionada ao uso de fármacos sem orientação veterinária. O estudo mostrou ser frequente o uso irracional de medicamentos em animais de companhia sem prescrição realizada pelo médico veterinário na população atendida pelo HCV/UFPEL.

Palavras-chave: Intoxicações. Medicamentos. Pequenos animais. 


\section{INTRODUÇÃO}

O Brasil atualmente possui 52,2 milhões de cães e 22,1 milhões de gatos, de acordo com a Pesquisa Nacional de Saúde realizada pelo Instituto Brasileiro de Geografia e Estatística (IBGE) em 2013, e é o 5ㅇ maior mercado pet do mundo (SOUZA JÚNIOR et al., 2016). Devido à grande proximidade dos animais de companhia com o ser humano e o papel que o pet desempenha no círculo familiar, ele está sujeito ao mesmo tratamento que os humanos (humanização), sendo submetido à prática da automedicação sem orientação profissional, o que configura uma das mais relevantes causas de intoxicações medicamentosas em cães e gatos (ANDRADE; NOGUEIRA, 2011; BISCHOFF; MUKAI, 2012; XAVIER et al., 2008).

Conforme a Agência Nacional de Vigilância Sanitária, a automedicação é caracterizada pela iniciativa de um doente, ou de seu responsável, em obter ou produzir e utilizar um produto que acredita lhe trará benefícios no tratamento de doenças ou alívio de sintomas, sem a orientação de um profissional de saúde qualificado (ANVISA, 2007).

Este trabalho visou determinar a prevalência dos casos de medicação de animais de companhia sem orientação do médico veterinário, bem como identificar os fármacos mais utilizados. Pretendia-se ainda identificar os potenciais causadores de toxicoses medicamentosas, assim como realizar uma breve revisão sobre a toxicidade destas drogas, de forma a estabelecer dados loco regionais referentes aos usuários do Hospital de Clínicas Veterinárias da Universidade Federal de Pelotas (HCV/UFPEL), situado na cidade de Capão do Leão - RS.

\section{MATERIAL E MÉTODOS}

A pesquisa foi conduzida na modalidade de levantamento, realizado a partir de questionário fechado disponibilizado em sala de espera do Hospital de Clínicas Veterinárias da Universidade de Pelotas (HCV/UFPEL), localizado na cidade de Capão do Leão - RS. Foram aplicados 267 questionários, sendo o responsável pelo paciente convidado a participar da pesquisa, com total liberdade para negar sua participação. Foram excluídos médicos veterinários e alunos do curso de graduação em Medicina Veterinária. Aos tutores participantes foi solicitado que assinassem o termo de consentimento livre e esclarecido relativo ao presente trabalho. Os dados coletados foram submetidos à análise descritiva. 
As questões abordadas no questionário incluíram o uso de medicações comerciais ou naturais sem orientação do médico veterinário, sua dosagem e frequência de uso, bem como a fonte da indicação do uso de tais medicamentos. Foram apresentadas questões de múltipla escolha, com a intenção de se identificar quais grupos de fármacos são mais comumente usados sem prescrição médico-veterinária, e os motivos pelo qual o fato ocorre. Além disso, pretendeu-se avaliar o envolvimento de leigos, a relevância da divulgação pela mídia, o alcance da internet e a atuação do vendedor de estabelecimentos agroveterinários.

Também foram indagados o grau de escolaridade do tutor, seu gênero e hábito de automedicar-se, buscando correlacionar o acesso à informação com a decisão da automedicação, bem como a predominância da cultura da automedicação e como ela alcança o animal de companhia no âmbito familiar.

Ao final, foram apresentadas afirmativas onde o tutor indica as opções que contêm fatos com os quais concorda. As questões abordadas foram relevantes na avaliação do perfil do tutor que pratica a medicação sem orientação profissional, e incluíram: a facilidade de adquirir medicamentos; a dificuldade de compreensão das prescrições médico veterinárias; o hábito de utilização de medicamentos e de receitas caseiras sem orientação profissional; o hábito de aplicar parasiticidas sem orientação profissional; a reutilização de prescrições antigas; o histórico de toxicoses medicamentosas causadas por automedicação em animais de companhia do tutor; a informação ao médico veterinário, durante a consulta, acerca de medicações utilizadas previamente sem sua orientação; o mito de que alguns fármacos são inócuos; a facilidade de acesso aos medicamentos armazenados na residência e o hábito de utilizar medicação com validade expirada.

Ao entregar o questionário preenchido, o tutor participante recebeu um material informativo que aborda de forma simples e concisa as principais causas de intoxicações em pequenos animais, os sinais clínicos mais frequentemente observados e a importância da orientação veterinária na terapêutica de animais de companhia.

O tamanho da amostra foi definido utilizando-se o cálculo amostral para população finita, a partir do número estimado de 800 atendimentos realizados em cães e gatos no HCV/UFPEL durante o ano de 2016, e objetivando nível de confiança de $95 \%$ e margem de erro de $5 \%$, 
com porcentagem de $50 \%$ para ocorrência do fenômeno. Este projeto foi aprovado pelo Comitê de Ética em Pesquisa (CEP) da Universidade Federal de Pelotas sob o parecer $\mathrm{n}^{\circ}$ 2.192.854.

\section{RESULTADOS E DISCUSSÃO}

A exposição de animais de companhia a fármacos tem aumentado devido ao maior uso desses agentes na medicina humana e veterinária nos últimos 20 anos. Embora se saiba que os medicamentos são a terceira maior causa de intoxicações em cães e gatos no país, sua real incidência pode estar sendo subestimada, uma vez que os eventos de toxicidade não são todos reportados aos serviços oficiais (GWALTNEY-BRANT, 2011, 2012).

De acordo com o Centro de Informação Toxicológica do Rio Grande do Sul (CIT/RS, 2014) os medicamentos foram responsáveis por $28,7 \%$ das exposições a agentes tóxicos relatadas em humanos e animais em 2014 no Estado. Os medicamentos estão entre as principais causas de intoxicação nos animais de companhia no Brasil, sendo os anti-inflamatórios nãoesteroidais (AINEs), os analgésicos, os antibióticos, os tranquilizantes e os antiparasitários as categorias mais citadas (ANDRADE; NOGUEIRA, 2011). Esses dados sugerem que a prática da utilização de medicamentos sem orientação profissional no país contribui para o uso irracional de medicamentos no âmbito da medicina veterinária e evidenciam a falta de dados loco regionais sobre a prevalência da medicação em animais de companhia sem acompanhamento médico-veterinário e as consequentes intoxicações iatrogênicas.

No Brasil ainda há poucos estudos acerca da administração de medicamentos sem orientação profissional na medicina veterinária (SOUZA JÚNIOR et al., 2016). Um levantamento de casos de intoxicações em cães e gatos atendidos na Faculdade de Veterinária da Universidade Federal Fluminense entre 2002 e 2008 concluiu que a principal causa foi medicamentosa, atribuída ao uso inadequado de fármacos sem orientação veterinária (MEDEIROS et al., 2009). Souza Júnior et al. (2016) encontraram uma prevalência de $88 \%$ ao investigarem as vendas de medicamentos veterinários sem prescrição em duas cidades brasileiras (Nanuque - MG e Ponto Belo - ES), e constataram que as classes mais vendidas foram vermífugos, anti-inflamatórios e antibióticos. Em um levantamento realizado no Hospital Veterinário da Universidade Federal de Minas Gerais, Santos et al. (2014) 
concluíram que os medicamentos mais frequentemente implicados em intoxicações em animais de companhia foram AINEs, praguicidas/antiparasitários, tranquilizantes e antibióticos. Issakowicz et al. (2010) realizaram um levantamento na Clínica Escola Veterinária da UNICENTRO entre os anos de 2006 e 2008, e constataram que 27\% dos felinos atendidos naquele período foram medicados pelos proprietários sem a devida orientação profissional.

No presente trabalho, foram coletados dados de 267 animais, sendo 198 caninos e 69 felinos, sob responsabilidade de 180 tutores. Os fármacos mais utilizados sem orientação profissional em caninos foram os pertencentes ao grupo dos ectoparasiticidas, os analgésicos e os anti-inflamatórios. Já em felinos, foi evidenciada maior utilização de antibióticos, analgésicos e anti-inflamatórios sem indicação veterinária. A prevalência dos grupos farmacológicos administrados a cães e gatos sem prescrição médico-veterinária está demonstrada nas Figuras 1 e 2.

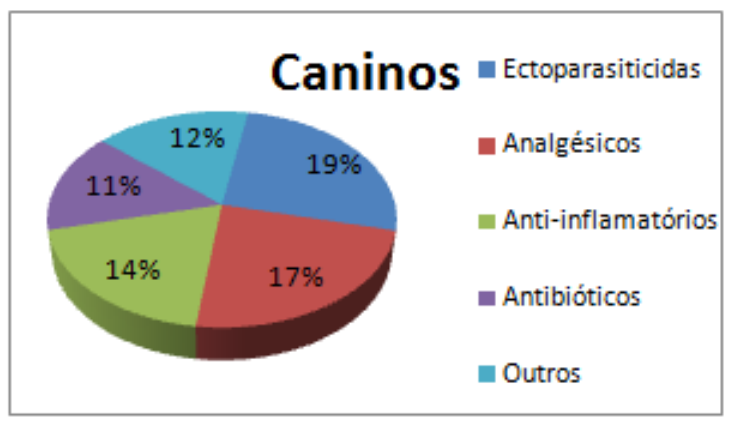

Figura 1 - Grupos farmacológicos administrados aos caninos sem orientação profissional, informados por usuários do Hospital de Clínicas Veterinárias da Universidade Federal de Pelotas.

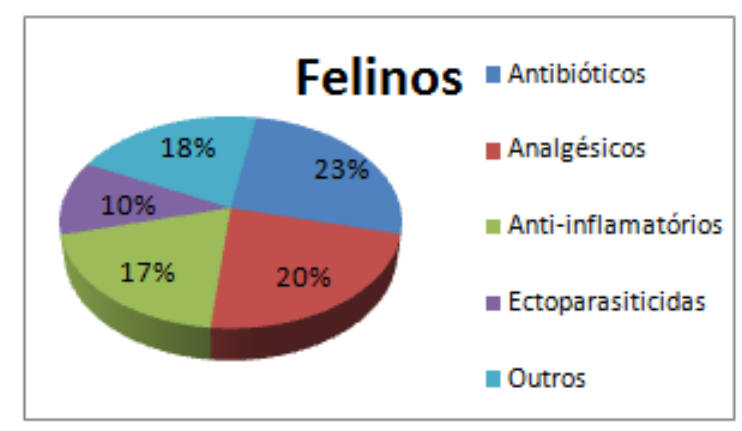

Figura 2 - Grupos farmacológicos administrados aos felinos sem orientação profissional, informados por usuários do Hospital de Clínicas Veterinárias da Universidade Federal de Pelotas. 
Quanto ao perfil dos tutores, $97 \%$ das mulheres e $75 \%$ dos homens declararam praticar a automedicação. Dentre as mulheres que se automedicam, 10\% possuem ensino fundamental, 31\% ensino médio, 38\% graduação e $20 \%$ pós-graduação. Em relação ao nível de escolaridade dos homens que se automedicam, $11 \%$ possuem ensino fundamental, $45 \%$ ensino médio, $27 \%$ graduação e $18 \%$ pós-graduação. Vinte e um por cento das mulheres e $19 \%$ dos homens que participaram deste estudo declararam medicar seus animais sem orientação profissional, com regularidade. Das mulheres que medicam seus pets sem orientação veterinária, $16 \%$ tem ensino fundamental, 32\% ensino médio, 36\% graduação e $16 \%$ pós-graduação. Dentre os homens, $18 \%$ possuem ensino fundamental, $64 \%$ ensino médio e $18 \%$ graduação. Estes dados comprovam a relação entre o hábito da automedicação e a prática do uso de fármacos sem orientação profissional em animais de companhia, e demonstram que a automedicação ocorre indistintamente entre tutores de ambos os sexos, estando de acordo com estudo de Menezes et al. (2008).

Quando indagados sobre o uso de medicamentos prévio à consulta no HCV/UFPEL, 20\% dos tutores relataram medicar seus animais de companhia sem orientação médico-veterinária, e $72 \%$ afirmaram comunicar ao veterinário que o animal foi previamente medicado. Nove por cento dos tutores já presenciaram toxicidade medicamentosa relacionada ao uso de fármacos sem orientação veterinária. O uso irracional de medicamentos é um problema mundial de saúde pública, e configura o uso desnecessário e equivocado de antibióticos, a prescrição excessiva de medicamentos por paciente, e a autoprescrição com fármacos de venda controlada (WCC, 2006). Grand et al. (1999) e Beyene e Tesega (2014) referem as intoxicações como principais consequências da automedicação.

O uso de fármacos em animais de companhia sem orientação profissional inclui a prescrição realizada por pessoas não qualificadas, o uso de formulações caseiras e a automedicação orientada através da reutilização de prescrições antigas, e é contraindicada pela impossibilidade de extrapolação da dose terapêutica dos fármacos entre humanos e animais (ANDRADE; NOGUEIRA, 2011; BISCHOFF; MUKAI, 2012; CARVALHO et al., 2012; DECLEMENTI, 2012; RECHE JÚNIOR; PIMENTA, 2012). Deve-se ressaltar a impossibilidade da extrapolação de regimes terapêuticos interespécies, sendo os principais exemplos dessa 
afirmativa o cão e o gato, devido a particularidades envolvendo os processos de distribuição e biotransformação de drogas em felinos (LITTLE; RICHARDSON, 2016; RECHE JÚNIOR; PIMENTA, 2012).

Anti-inflamatórios foram administrados a $14 \%$ dos cães sem prescrição veterinária atual. Os princípios utilizados foram flunixin meglumine, carprofeno, meloxicam, ibuprofeno, diclofenaco e cetoprofeno. Dezessete por cento dos gatos receberam anti-inflamatórios sem recomendação veterinária, sendo citados: cetoprofeno, meloxicam e ibuprofeno. O Brasil é o 9o consumidor mundial de AINEs, e as intoxicações em pequenos animais ocorrem pela ansiedade do tutor em aliviar os sintomas, ou pelo prolongamento do tratamento prescrito. Os animais são mais sensíveis aos AINEs do que os humanos, e grande parte das intoxicações por esta classe medicamentosa se deve à utilização de fármacos sem orientação profissional (RIBOLDI et al., 2012).

Analgésicos e antitérmicos foram administrados a 17\% dos cães na ausência de prescrição veterinária atual. Os princípios utilizados incluíram a dipirona, o tramadol e o paracetamol. Vinte por cento dos felinos receberam dipirona sem prescrição veterinária atualizada. Segundo Siroka e Svobodova (2013) a dipirona é metabolizada lentamente nesta espécie, podendo causar intoxicações quando utilizada em doses elevadas ou intervalos reduzidos. Importante ressaltar ainda que os gatos são mais suscetíveis à intoxicação por paracetamol em comparação a outras espécies, devido a sua baixa capacidade de glicuronidação, o que dificulta a eliminação de seus metabólitos. Nesta espécie ocorre a formação de um metabólito altamente reativo capaz de produzir severo dano oxidativo ao ferro dos eritrócitos, podendo causar metahemoglobinemia (BISCHOFF; MUKAI, 2012; SIROKA; SVOBODOVA, 2013). A causa mais comum de intoxicação por paracetamol em cães e gatos é a administração por iniciativa do tutor, sem orientação veterinária (BISCHOFF; MUKAI, 2012; CORTINOVIS et al., 2015). Cães são mais sensíveis ao ibuprofeno, ao flunixin meglumine, ao diclofenaco e ao naproxeno devido à circulação enterohepática que estes fármacos sofrem na espécie, prolongando sua meia-vida plasmática. Doses maiores que $5 \mathrm{mg} / \mathrm{kg}$ de ibuprofeno já são associadas com efeitos colaterais em cães, fato preocupante quando consideramos que as apresentações comerciais no Brasil contêm até $600 \mathrm{mg}$ do princípio 
ativo (BISCHOFF; MUKAI, 2012; CORTINOVIS et al., 2015). Os efeitos colaterais mais comumente associados ao uso de AINEs são insuficiência renal e ulceração gastrointestinal, porém também são relatados casos de ceratoconjuntivite seca em cães e hematotoxicidade (BISCHOFF; MUKAI, 2012; HAMPSHIRE et al., 2004; SIROKA; SVOBODOVA, 2013).

Seis por cento dos cães e $3 \%$ dos gatos receberam glicocorticoides sem recomendação veterinária atual. Em felinos, o uso da prednisolona é preferível ao da prednisona, em função da sua farmacocinética superior nesta espécie (BLOIS; MATHEWS, 2016). Efeitos colaterais significativos são associados à terapia em longo prazo ou com altas doses, e incluem ulceração gastrointestinal, pancreatite, hepatopatia, glomerulopatia, hiperadrenocorticismo iatrogênico, hipercoagulabilidade, imunossupressão, insuficiência cardíaca congestiva e diabetes mellitus, estes dois últimos especialmente em felinos (DAY, 2010; PLUMB, 2011).

Quanto ao uso de antibióticos, $11 \%$ dos cães estavam sendo tratados com fármacos desta classe sem prescrição atual de médico veterinário. Os antimicrobianos utilizados foram amoxicilina associada ou não ao clavulanato de potássio, enrofloxacina, norfloxacina, associação comercial de penicilinas com dihidroestreptomicina, cefalexina, tetraciclina, sulfadiazina com trimetoprim, doxiciclina e rifampicina. Já em relação aos felinos, 23\% receberam antibióticos sem prescrição veterinária atual. Os princípios relacionados foram amoxicilina, enrofloxacina, associação de penicilinas com dihidroestreptomicina, cefalotina, oxitetraciclina e sulfadiazina com trimetoprim.

O uso irracional de antimicrobianos é um problema atual em todo o mundo, especialmente em países em desenvolvimento onde é possível a venda de antibióticos sem retenção de receita (BENNADI, 2014). A principal preocupação acerca do uso irracional de antibióticos é o desenvolvimento da resistência microbiana, com sérias implicações tanto na medicina humana quanto na medicina veterinária (BEYENE; TESEGA, 2014). Entretanto, o uso inadequado de antimicrobianos também favorece o supercrescimento de micro-organismos não sensíveis, aumentando o risco de infecções oportunistas (SOUSA, 2006). As fluorquinolonas podem induzir artropatias em animais jovens (XAVIER et al., 2008). A enrofloxacina foi implicada em casos de cegueira aguda por degeneração de retina em felinos (HAMPSHIRE et al., 2004). Sulfonamidas podem causar efeitos tóxicos agudos e 
crônicos, destacando a cristalúria, a anemia aplásica e as reações de hipersensibilidade. A maioria dos efeitos colaterais das penicilinas está relacionada às reações de hipersensibilidade (MADDISON et al., 2010). As tetraciclinas podem causar hepatotoxicidade quando em doses elevadas. Reações adversas às cefalosporinas são incomuns e, quando ocorrem, geralmente se restringem a vômitos e diarreia (MADDISON et al., 2010; XAVIER et al., 2008).

Quanto ao uso de ectoparasiticidas, $19 \%$ dos caninos foram tratados com esta classe medicamentosa sem acompanhamento do médico veterinário. Os produtos utilizados envolveram deltametrina, cipermetrina, benzoato de benzila 10\%, nitempiram, fipronil, amitraz 12,5\%, moxidectina 1\%, associação de clorpirifós, cipermetrina e fenthion, associação de trissulfeto de arsênico, enxofre sublimado e alcatrão (sabão medicinal), associação de ácido bórico, permetrina e óxido de zinco (unguento) e associação de sulfadiazina prata, alumínio, permetrina e diclorvós (spray). Dez por cento dos gatos receberam ectoparasiticidas sem prescrição veterinária atual. Os princípios utilizados foram deltametrina e fipronil. Sessenta e três por cento dos tutores participantes deste trabalho declararam aplicar antiparasitários nos seus animais de companhia sem acompanhamento veterinário. O mau uso de parasiticidas veterinários é uma causa comum de intoxicação medicamentosa em cães e gatos (CALONI et al., 2012).

O fipronil é um antiparasitário considerado seguro para cães e gatos, porém casos de toxicidade continuam sendo relatados, envolvendo alopecia local no sítio de administração, prurido e sinais neurológicos. O amitraz é um acaricida com efeitos adversos comuns a todos os mamíferos, causando em gatos severa depressão respiratória e hipotermia (SIROKA; SVOBODOVA, 2013). Esta espécie é extremamente sensível à intoxicação por algumas piretrinas e piretróides, devido à deficiência de metabolização através da glicuronidação e ao hábito de praticar grooming (BOLAND; ANGLES, 2010; CALONI et al., 2014; GWALTNEYBRANT, 2012; SUTTON et al., 2007).

Em relação ao uso da ivermectina, 3,5\% dos cães e 1,5\% dos gatos haviam recebido este fármaco sem qualquer indicação veterinária. Siroka e Svobodova (2013) contraindicam o uso 
da ivermectina em animais de companhia, já que intoxicações têm sido descritas em muitas raças de cães e em gatos.

Complexos vitamínicos e estimulantes do apetite foram administrados a $2 \%$ dos cães e $7 \%$ dos gatos sem indicação veterinária. Dois cães e um gato receberam suplementação de vitamina D de uso humano. Conforme Schell e Gwaltney-Brant (2011), a ingestão de vitamina $D$ é uma preocupação quando ultrapassa $4.000 \mathrm{Ul} / \mathrm{kg}$, pois a sobredose causa aumento do cálcio e do fósforo séricos e sua consequente deposição nas paredes vasculares, levando a rigidez vascular e, potencialmente, à morte súbita.

Quanto aos tratamentos naturais, $6 \%$ dos cães e $12 \%$ dos felinos receberam chás, sendo citados: boldo, tanchagem, quebra-pedra, hortelã, sene, losna, macela, camomila, cavalinha e anis. Dez por cento dos tutores declararam administrar receitas caseiras aos seus animais habitualmente. De acordo com Leite et al. (2006) e Silva Filho et al. (2013), a administração de remédios caseiros à base de ervas ou vegetais potencialmente tóxicos frequentemente levam animais ao óbito.

Um canino recebeu clonazepam, um benzodiazepínico amplamente utilizado em medicina humana para tratamento da ansiedade, distúrbios do sono e crises de pânico. Segundo Cortinovis et al. (2015) os sinais clínicos da toxicose em pequenos animais incluem ataxia, sedação, tremores, vômitos, hipotermia, coma e depressão respiratória. Efeitos incomuns são hiperestesia, hipersalivação, agitação, agressividade e hipertermia.

Um canino foi sedado sem acompanhamento veterinário, e um foi medicado com diazepam. Cinco cães receberam laxantes, um cão recebeu óleo queimado e graxa por via tópica e em um felino foi aplicado iodo tópico. Quatro cadelas e duas gatas receberam progestágeno, administrado pelo balconista de casa comercial de produtos veterinários. Os progestágenos são comumente utilizados como contraceptivos, no intuito de prevenir o retorno ao ciclo estral e inibir as ovulações, entretanto, seus efeitos colaterais fizeram com que sua utilização em pequenos animais seja contraindicada. Em cadelas e gatas o acetato de megestrol pode causar supressão adrenocortical e atrofia da adrenal, diabetes mellitus transitório, sobrepeso, hiperplasia endometrial cística, piometra, neoplasia mamária e 
hepatotoxicidade. Em gatas, é relacionado ainda com a hiperplasia fibroepitelial mamária (NARDI et al., 2008; THOMAS; FONTBONE, 2010).

Arrais et al. (1997) afirmaram que a escolha dos medicamentos implicados na automedicação é baseada principalmente na recomendação de leigos e na reutilização de prescrições anteriores, fato comprovado por este trabalho. A indicação do uso dos medicamentos descritos partiu: em 7\% dos casos, de um amigo; em 9\% dos casos, de um familiar; em $20 \%$ dos casos, do próprio tutor; e em $31 \%$ dos casos, do balconista. Em $21 \%$ dos casos a fonte dos medicamentos foi uma prescrição médico-veterinária anterior, e em 8\% dos casos os animais estavam sendo medicados a partir de uma prescrição veterinária atual. Quatro por cento dos tutores optaram por não responder a esta pergunta. Apenas $1 \%$ dos tutores foi influenciado por propagandas e $3 \%$ pela internet, dados que corroboram a afirmativa da World Self-Medication Industry (WSMI, 2008) de que a mídia não parece influenciar de maneira significativa o consumo de medicamentos pela população em geral, porém a internet está emergindo como a maior fonte de informações relativas à saúde.

A predominância da figura do balconista prescritor é extremamente preocupante, uma vez que favorece o uso inadequado dos medicamentos, a prática da "empurroterapia", a perpetuação da crença de que não há saúde sem utilização de medicamentos e o subdiagnóstico de zoonoses (BORTOLON et al., 2007; FVE, 1999; HAAK, 1989; MELO et al., 2006; SOUSA, 2006; VITOR et al., 2008). Adicionalmente, o risco de infecção por injeções aplicadas com pouca atenção à antissepsia ou sem recomendação é uma preocupação constante (MARIN, 2003).

Dentre os motivos que levaram os tutores a medicar seus animais sem orientação do médico veterinário, a tentativa de resolver o problema em casa foi a principal razão, com $39 \%$ dos casos. Segundo Fernandes e Cembranelli (2015) febre e dor são os principais indutores da automedicação humana, e podemos inferir que os sintomas apresentados pelos animais de companhia são interpretados como sendo febre ou dor pelos tutores, levando à administração bem-intencionada de medicamentos de venda livre (BISCHOFF; MUKAI, 2012). Vinte e seis por cento dos tutores declararam haver tido boas experiências anteriores com o medicamento. Conforme o World Council of Churches (WCC, 2006), a utilização de 
medicamentos receitados para o tratamento de um paciente e utilizados em outro indivíduo da mesma família é um clássico exemplo de uso irracional. Oito por cento declararam restrições financeiras que os impediam de procurar o serviço médico-veterinário, e $1 \%$ declarou conhecer melhor o seu animal do que o médico veterinário, corroborando a afirmação de Bennadi (2014) de que a empatia pelo paciente é um dos fatores que predispõe à automedicação. Vinte e seis por cento dos tutores declararam não ter justificativa para medicar seus animais sem orientação veterinária.

Seis por cento dos tutores participantes deste trabalho declararam ter dificuldade em compreender a prescrição veterinária, dado discrepante quando comparado ao divulgado pela World Health Organization (VRIES et al., 1996), pois mais de $40 \%$ dos pacientes entrevistados não compreenderam a prescrição médica. De acordo com Homedes e Ugalde (1993) e Oliveira (2008), o entendimento da prescrição é um pré-requisito para a adesão ao tratamento, sendo esta a etapa final do uso racional de medicamentos. Segundo Kelly (1994) a falta de compreensão acerca do regime terapêutico leva a não adesão ao tratamento duas vezes mais frequentemente do que o abandono voluntário.

Vinte e dois por cento dos tutores participantes deste trabalho acreditam que alguns medicamentos são inofensivos e é possível utilizá-los por conta própria, e 58\% consideram muito fácil adquirir medicamentos sem orientação profissional. A World Health Organization alerta que nenhuma droga é absolutamente segura, todo medicamento apresenta risco, e ele aumenta com o uso irracional (WHO, 2000). Portanto, a recomendação para o uso de fármacos em animais, deve partir tão e somente de um médico veterinário.

\section{CONCLUSÃO}

O presente estudo concluiu que é frequente o uso indiscriminado de medicamentos em animais de companhia sem prescrição realizada por médico veterinário, sendo que o uso de fármacos em animais de companhia sem recomendação veterinária ocorre indistintamente entre tutores de ambos os sexos. Os ectoparasiticidas, antibióticos, analgésicos e antiinflamatórios foram os principais grupos medicamentosos administrados pelos tutores. Concluímos também que, independentemente dos motivos para realizar a automedicação, os tutores têm ciência da ocorrência de intoxicações decorrentes deste procedimento. 


\section{EVALUATION OF THE USE OF DRUGS IN COMPANION ANIMALS WITHOUT PROFESSIONAL GUIDANCE}

\section{ABSTRACT}

$\mathrm{T}$ he use of drugs in companion animals without professional guidance includes prescription by unqualified people, use of home-made formulations, and reuse of old veterinarian prescriptions. This work aims to determine the prevalence of pet medication cases without the veterinarian's guidance, as well as to identify the most used drugs, establishing loco regional data referring to the users of the Veterinary Hospital of the Federal University of Pelotas (HCV/UFPEL) located in the city of Capão do Leão - RS. The research was carried out from a closed questionnaire made available in the waiting room of the HCV/UFPEL, and the questions addressed included the use of commercial or natural medications without the veterinarian's instructions, the indication of lay people and the tutors' profile. Data were collected from 267 animals, being 198 canines and 69 felines, under the responsibility of 180 tutors. Twenty-one percent of women and $19 \%$ of men medicate their animals without professional guidance on a regular basis. Seventy-two percent of the tutors stated that they reported to the veterinarian that the animal had previously been medicated, and $9 \%$ had already seen drug toxicity related to the use of drugs without veterinary guidance. The irrational use of medicines in companion animals is frequent without veterinary prescription.

Keywords: Intoxications. Medicines. Small animals.

\section{EVALUACIÓN DEL USO DE FÁRMACOS EN ANIMALES DE COMPAÑÍA SIN ORIENTACIÓN PROFESIONAL}

\section{RESUMEN}

$\mathrm{E}$ uso de fármacos en animales de compañía sin orientación profesional incluye la prescripción realizada por personas no cualificadas, el uso de formulaciones caseras y la automedicación orientada - cuándo el tutor reutiliza prescripciones antiguas. Este trabajo pretendió determinar la prevalencia de los casos de medicación de animales de compañía sin orientación del médico veterinario, así como identificar los fármacos más utilizados, estableciendo datos loco regionales referentes a los usuarios del Hospital de Clínicas Veterinarias de la Universidad Federal de Pelotas (HCV/UFPEL), ubicado en la ciudad de Capão do Leão - RS. Los datos fueron obtenidos a partir de un cuestionario confidencial 
disponible en sala de espera del HCV/UFPEL, y las cuestiones abordadas incluyeron el uso de medicamentos comerciales o naturales sin orientación del médico veterinario, la indicación de laicos y el perfil de los tutores. Se recogieron datos de 267 animales, siendo 198 caninos y 69 felinos, bajo la responsabilidad de 180 tutores. Veinte y uno por ciento de las mujeres y el $19 \%$ de los hombres medican a sus animales sin orientación profesional con regularidad. Setenta y dos por ciento de los tutores afirmó comunicar al veterinario que el animal fue previamente medicado, y el $9 \%$ ya presenció toxicidad medicamentosa relacionada con el uso de fármacos sin orientación veterinaria. Es frecuente el uso irracional de medicamentos en animales de compañía sin prescripción realizada por el médico veterinario.

Palabras clave: Intoxicaciones. Drogas. Pequeños animales.

\section{REFERÊNCIAS}

ANDRADE, S. F.; NOGUEIRA, R. M. B. Toxicologia veterinária. São Paulo: Roca, 2011. P. 243272.

ANVISA - AGÊNCIA NACIONAL DE VIGILÂNCIA SANITÁRIA. Caderno do Professor - Projeto Educação e Promoção da Saúde no Contexto Escolar: o contributo da Agência Nacional de Vigilância Sanitária para o uso racional de medicamentos. Brasília: GPROP/DIFRA, 2007. 80p. Disponível em:

<http://www.anvisa.gov.br/propaganda/educacao_saude/caderno_professor.pdf>.

ARRAIS, P. S. D.; COELHO, H. L. L.; BATISTA, M. C. D. S.; et al. Perfil da automedicação no Brasil. Revista de Saúde Pública, v. 31, n. 1, p. 71-77, 1997.

BENNADI, D. Self-medication: a current challenge. Journal of Basic and Clinical Pharmacy, v. 5, n. 1, p. 19-23, 2014.

BEYENE, T.; TESEGA, B. Rational veterinary drug use: its significance in public health. Journal of Veterinary Medicine and Animal Health, v. 6, n. 12, p. 302-308, 2014. Disponível em: <http://www.academicjournals.org/app/webroot/article/article1417096829_Beyene\%20an d\%20Tesega.pdf>.

BISCHOFF, K.; MUKAI, M. Toxicity of over-the-counter drugs. In: GUPTA, R. C. Veterinary Toxicology - Basic and Clinical Principles. 2. ed. Elsevier, 2012. P. 443-468.

BLOIS, S.; MATHEWS, K. A. Anti-inflammatory therapy. In: ETTINGER, S. J.; FELDMAN, E. C.; CÔTÉ, E. Textbook of Veterinary Internal Medicine. 8. ed. Elsevier, 2016. P. 1782-1793.

BOLAND, L. A.; ANGLES, J. M. Feline permethrin toxicity: retrospective study of 42 cases. Journal of Feline Medicine and Surgery, v. 12, n. 2, p. 61-71, 2010. 
BORTOLON, P. C.; KARNIKOWSKI, M. G. O.; ASSIS, M. Automedicação versus indicação farmacêutica: o profissional de farmácia na atenção primária à saúde do idoso. Revista de Atenção Primária à Saúde, v. 10, n. 2, p. 200-209, 2007.

CALONI, F.; CORTINOVIS, C.; PIZZO, F.; et al. Epidemiological study (2006-2012) on the poisoning of small animals by human and veterinary drugs. Veterinary Record, v. 174, n. 9, p. 222, 2014.

CALONI, F.; CORTINOVIS, C.; RIVOLTA, M.; et al. Animal poisoning in Italy: 10 years of epidemiological data from the poison Control Centre of Milan. Veterinary Record, v. 170, n. 16, p. 415, 2012.

CARVALHO, C. F.; ARAÚJO, D. P.; BONFIM, J. C.; et al. Incidência de medicação em cães e gatos por seus responsáveis sem orientação médico-veterinária: levantamento em um hospital veterinário universitário. Enciclopédia Biosfera, v. 8, n. 15, p. 1035-1042, 2012.

CIT/RS - CENTRO DE INFORMAÇÃO TOXICOLÓGICA DO RIO GRANDE DO SUL. Relatório Anual de Atendimento - 2014. Porto Alegre: CIT/RS, 2014. 49p. Disponível em:

<https://drive.google.com/file/d/OB8zsofysQ_DTQmlwNG5mY3NTMk0/view>.

CORTINOVIS, C.; PIZZO, F.; CALONI, F. Poisoning of dogs and cats by drugs intended for human use. The Veterinary Journal, v. 203, n. 1, p. 52-58, 2015.

DAY, M. J. Glicocorticosteroides e anti-histamínicos. In: MADDISON, J. E.; PAGE, S. W.; $\mathrm{CHURCH}, \mathrm{D}$. B. Farmacologia clínica de pequenos animais. Rio de Janeiro: Elsevier, 2010. P. 257-265.

DECLEMENTI, C. Prevention and treatment of poisoning. In: GUPTA, R. C. Veterinary Toxicology - Basic and Clinical Principles. 2. ed. Elsevier, 2012. P. 1361-1379.

FVE - FEDERATION OF VETERINARIANS OF EUROPE. Antibiotic Resistance \& Prudent use of Antibiotics in Veterinary Medicine. Brussels, 1999. 12p. Disponível em: $<$ http://www.fve.org/news/publications/pdf/antibioen.pdf>.

FERNANDES, W. S.; CEMBRANELLI, J. C. Automedicação e o uso irracional de medicamentos: o papel do profissional farmacêutico no combate a essas práticas. Revista Univap, v. 21, n. 37, p. 5-12, 2015.

SILVA FILHO, M. P.; COELHO, I. D. S.; LIMA, R. P.; et al. Indicações de medicamentos de uso veterinário por balconistas de farmácias e estabelecimentos veterinários em diversos municípios do Estado da Paraíba. Revista de Biologia e Farmácia, v. 9, n. 3, p. 1-5, 2013.

GRAND, A.; HOGERZEIL, H. V.; HAAIJER-RUSKAMP, F. M. Intervention research in rational use of drugs: a review. Health Policy and Planning, n. 14, v. 2, p. 89-102, 1999. 
GWALTNEY-BRANT, S. M. Incidence of poisoning in small animals. In: POPPENGA, R. H.; GWALTNEY-BRANT, S. M. Small Animals Toxicology Essentials. Wiley-Blackwell, 2011. P. 1720.

GWALTNEY-BRANT, S. M. Epidemiology of animal poisonings in the United States. In: GUPTA, R. C. Veterinary Toxicology - Basic and Clinical Principles. 2. ed. Elsevier, 2012. P. 80-87.

HAAK, H. Padrões de consumo de medicamentos em dois povoados da Bahia (Brasil). Revista de Saúde Pública, São Paulo, v. 23, n. 2, p. 143-151, 1989.

HAMPSHIRE, V. A.; DODDY, F. M.; POST, L. O.; et al. Adverse drug event reports at the United States Food and Drug Administration Center for Veterinary Medicine. Journal of the American Veterinary Medical Association, v. 225, n. 4, p. 533-536, 2004.

HOMEDES, N.; UGALDE, A. Patients' compliance with medical treatments in the third world. What do we know? Health Policy and Planning, v. 8, n. 4, p. 291-314, 1993.

IBGE - INSTITUTO BRASILEIRO DE GEOGRAFIA E ESTATÍSTICA. Pesquisa Nacional de Saúde 2013. Rio de Janeiro: IBGE, 2015. 100p. Disponível em: <https://biblioteca.ibge.gov.br/visualizacao/livros/liv94074.pdf>.

ISSAKOWICZ, J. C.; NICOLAO, T. C.; VIEIRA, M. N.; et al. Casuística dos atendimentos de felinos na Clínica Escola Veterinária (CEVET) da UNICENTRO no triênio 2006-2008. Revista Científica Eletrônica de Medicina Veterinária, v. 8, n. 14, p. 1-6, 2010.

KELLY, J. M. Implementing a patient self-medication program. Rehabilitation Nursing, v. 19, n. 2, p. 87-90, 1994.

LEITE, L. C.; VILLANOVA JÚNIOR, J. A.; CIRIO, S. M.; et al. Prescrição de medicamentos veterinários por leigos: um problema ético. Revista Acadêmica Ciência Animal, v. 4, n. 4, p. 43-47, 2006.

LITTLE, S. E.; RICHARDSON, J. A. Toxicologia. In: LITTLE, S. E. O gato: medicina interna. Rio de Janeiro: Roca, 2016. P. 880-899.

MADDISON, J. E.; WATSON, D. J.; ELLIOTT, J. Medicamentos antibacterianos. In: MADDISON, J. E.; PAGE, S. W.; CHURCH, D. B. Farmacologia clínica de pequenos animais. Rio de Janeiro: Elsevier, 2010. P. 147-184.

MARIN, N.; LUIZA, V. L.; CASTRO, C. G. S. O.; SANTOS, S. M. (Org.). Assistência farmacêutica para gerentes municipais. Rio de Janeiro: OPAS/OMS, 2003. 373p. Disponível em:

$<$ http://www.cff.org.br/userfiles/84\%20-

\%20MARIN\%20N\%20ET\%20AL\%20Assistencia\%20Farmaceutica\%20para\%20gerentes\%20mu nicipais_2003.pdf>. 
MEDEIROS, R. J.; MONTEIRO, F. O.; SILVA, G. C.; et al. Casos de intoxicações exógenas em cães e gatos atendidos na Faculdade de Veterinária da Universidade Federal Fluminense durante o período de 2002 a 2008. Ciência Rural, Santa Maria, v. 39, n. 7, p. 2105-2110, 2009.

MELO, D. O.; RIBEIRO, E.; STORPIRTIS, S. A importância e a história dos estudos de utilização de medicamentos. Revista Brasileira de Ciências Farmacêuticas, v. 42, n. 4, p. 475-485, 2006.

MENEZES, E. A.; MESQUITA, J. L. S.; TEIXEIRA, A. B.; et al. Automedicação com antimicrobianos para infecções respiratórias na cidade de Fortaleza - CE. Infarma, v. 20, n. 7/8, p. 3-7, 2008.

NARDI, A. B.; RODASKI, S.; ROCHA, N. S.; et al. Neoplasias mamárias. In: DALECK, C. R.; NARDI, A. B.; RODASKI, S. Oncologia em Cães e Gatos. São Paulo: Roca, 2008. P. 371-383.

OLIVEIRA, E. A. Adesão à terapêutica medicamentosa. Infarma, v. 20, n. 7-8, p. 38-43, 2008.

PLUMB, D. C. Veterinary drug handbook. 7. ed. PharmaVet Inc: 2011.

RECHE JÚNIOR, A.; PIMENTA, M. M. Aspectos diferenciais no uso de fármacos em felinos. In: RABELO, R. C. Emergências em pequenos animais: condutas clínicas e cirúrgicas no paciente grave. Rio de Janeiro: Elsevier, 2012. P. 583-590.

RIBOLDI, E.; LIMA, D. A.; DALLEGRAVE, E. Sensibilidade espécie-específica dos antiinflamatórios não esteroidais: humanos $X$ animais de companhia. Arquivo Brasileiro de Medicina Veterinária e Zootecnia, v. 64, n. 1, p. 39-44, 2012.

SANTOS, W. G.; MARTINS, G. C.; MELO, M. M.; et al. Intoxicação por medicamentos em cães e gatos atendidos no Hospital Veterinário da UFMG. In: CONGRESSO BRASILEIRO DA

ANCLIVEPA, 35, 2014, Belo horizonte. ANAIS. Belo Horizonte, 2014. P. 1080-1082. Disponível em:

<http://www.infoteca.inf.br/anclivepa/smarty/templates/arquivos_template/upload_arquiv os/docs/ANC14350.pdf>.

SCHELL, M. M.; GWALTNEY-BRANT, S. M. OTC drugs. In: POPPENGA, R. H.; GWALTNEYBRANT, S. M. Small animals toxicology essentials. Wiley-Blackwell, 2011. P. 231-239.

SIROKA, Z.; SVOBODOVA, Z. The toxicity and adverse effects of selected drugs in animals overview. Polish Journal of Veterinary Sciences, v. 16, n. 1, p. 181-191, 2013.

SOUSA, F. F. O. Perfil da automedicação com antimicrobianos em farmácias de Fortaleza. Fortaleza, UFC, 2006, 137p. Dissertação (Mestrado em Ciências Farmacêuticas), Faculdade de Farmácia, Odontologia e Enfermagem, Departamento de Farmácia, Universidade Federal do Ceará, 2006. 
SOUZA JÚNIOR, L. O.; BREMER, D. K. C.; SOUZA, K. P.; et al. Panorama do comércio de medicamentos veterinários sem receita em lojas de produtos agropecuários nas cidades de Nanuque/MG e Ponto Belo/ES, e os perigos que esse fato pode acometer à saúde pública. In: REDES SOCIAIS E APRENDIZAGEM: REINVENTANDO O CONHECIMENTO, 2016, Porto Seguro. RESUMOS. Porto Seguro, 2016. Disponível em: <https://docplayer.com.br/39314022Laureano-orneles-de-souza-junior-graduando-em-farmacia-centro-universitario-decaratinga-campus-unec-de-nanuque.html>.

SUTTON, N. M.; BATES, N.; CAMPBELL, A. Clinical effects and outcome of feline permethrin spot-on poisonings reported to the Veterinary Poisons Information Service (VPIS), London. Journal of Feline Medicine and Surgery, v. 9, n. 4, p. 335-339, 2007.

THOMAS, P. G. A.; FONTBONE, A. Medicamentos e reprodução. In: MADDISON, J. E.; PAGE, S. W.; $\mathrm{CHURCH,} \mathrm{D.} \mathrm{B.} \mathrm{Farmacologia} \mathrm{clínica} \mathrm{de} \mathrm{pequenos} \mathrm{animais.} \mathrm{Rio} \mathrm{de} \mathrm{Janeiro:} \mathrm{Elsevier,}$ 2010. P. 520-537.

VITOR, R. S.; LOPES, C. P.; MENEZES, H. S.; et al. Padrão de consumo de medicamentos sem prescrição médica na cidade de Porto Alegre - RS. Ciência \& Saúde Coletiva, v. 13 (Sup), p. 737-743, 2008.

VRIES, T. P. G. M.; HENNING, R. H.; HOGERZEIL, H. V.; FRESLE, D. A. Guide to good prescribing: a practical manual. Geneva: World Health Organization 1996. 142p. Disponível em: <http://apps.who.int/medicinedocs/pdf/whozip23e/whozip23e.pdf>.

WCC - WORLD COUNCIL OF CHURCHES. Promoting rational use of medicines. Contact, $\mathrm{n}$. 183, p. 1-32, 2006. Disponível em:

<http://apps.who.int/medicinedocs/documents/s19836en/s19836en.pdf>.

WHO - WORLD HEALTH ORGANIZATION. General Policy Issues. WHO Drug information, v. 14, n. 1, p. 1-2, 2000. Disponível em:

<http://apps.who.int/medicinedocs/pdf/h1462e/h1462e.pdf>.

WSMI - WORLD SELF-MEDICATION INDUSTRY. Advertising of nonprescription medicines to the public, a significant contributor to healthcare. Ferney-Voltaire, 2008. 32p. Disponível em: <http://www.wsmi.org/wp-content/data/pdf/wsmi_brochureadvertising.pdf>.

XAVIER, F. G.; MARUO, V. M.; SPINOSA, H. S. Toxicologia dos medicamentos. In: SPINOSA, H. S. Toxicologia aplicada à medicina veterinária. Barueri: Manole, 2008. P. 117-189.

Autor para correspondência: Marta Zielke. Rua Dr. Bruno Chaves, n. 345, apto. 404 B, Três Vendas, Pelotas/RS, CEP: 96.055-040. martazielkevet@gmail.com.br 